\title{
HIV-1 Nef: hacker of the host cell
}

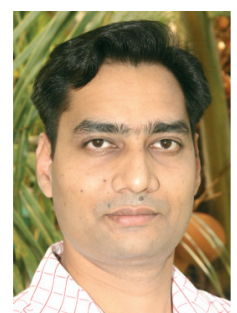

"Studies are still being carried out to understand

the fine mechanisms used by Nef to interact

with various cellular proteins that result in host

cell arrest, which make Nef so aggressive and

make HIV an effective virus."

\section{Shailendra K Saxena*1, Gaurav Shrivastava', Sneham Tiwari' \& Madhavan PN Nair ${ }^{2}$ \\ CSIR-Centre for Cellular \& Molecular Biology, Upoal Road, Hyderabad 500007 (AP), India \\ ${ }^{2}$ College of Medicine, Florida International University, Miami, FL 33199, USA \\ *Author for correspondence: Tel.: +91 4027192630 - Fax: +914027160591 = shailen@ccmb.res.in}

HIV-1 is a deadly virus that causes AIDS and is responsible for devastating lives globally over the last three decades [1]. HIV-1 has three structural genes ( $g a g$, pol and env), two regulatory genes (tat and $r e v$ ) and four accessory genes (vif, $v p u, v p r$ and nef). These work together to invade and degrade the host cell machinery, causing fatal disease. Of this group, one important gene is nef (which codes for the Nef protein), which has been an important focus for studies since 1988 (FIgUre 1). Initially, Nef was thought to play a negative role, possibly inhibiting viral replication, but later studies have shown it to be necessary for enhancing viral loadassociated infection, and inhibiting infected cell death. It has been shown that HIV-1 Nef plays a vital role in CD4 downregulation, MHC-I downregulation, CD28 downregulation and T-cell activation, which eventually contribute to disease progression [2]. Studies are still being carried out to understand the fine mechanisms used by Nef to interact with various cellular proteins that result in host cell arrest, which make Nef so aggressive and make HIV an effective virus.

\section{Introduction to HIV-1 Nef}

Nef is a tiny (206-amino acid) myristoylated protein that expresses early in the viral replication cycle and has a significant role in viral replication and pathogenesis. Even though no significant enzymatic functions of Nef have been reported, it is well-known to have interaction with several host cellular proteins (e.g., Pak2, Vav and Rac) [3]. Experiments using mouse models and Rhesus macaques have proved the importance of Nef, as models infected with Nef-deleted mutants did not display the typical disease progression towards clinical AIDS. Nef alters cellular pathways by acting as a multifarious molecular adaptor protein, with multiple conformations. It is a manipulator of protein trafficking, signal transduction cascades and apoptotic pathways that are mediated by a symphony of protein interactions with host cell factors. It modulates components of the endocytic sorting and T-cell receptor signaling machineries, and also host adapter proteins like Vav, Pak-2, Rac, CDC42 and the DOCK2-ELMO1 complex, resulting in alteration of signal transduction pathways [4]. Nef also inhibits apoptosis by modulating p53- and ASK1involved apoptotic pathways [5]. Nef removes CD4 from the cell surface, which may interfere with viral budding. It also binds to Gag-Pol and AIPI (a critical intermediate in the formation of multivesicular bodies), which contributes to the egress of viral particles from already-infected cells, such as via budding. Nef removes MHC-I from the cell surface, which makes it impossible for the cytotoxic T lymphocytes (CTLs) to recognize infected cells via peptides presented on MHC-I [6]. The downregulation of MHC-I decreases the efficiency of killing of HIV-1infected cells by CTLs [7]. Nef expression in early viral life cycle stages contributes to the two most important attributes of HIV-1 infection; T-cell activation and the maintenance of a stable state of infection. Its absence may completely prevent the development of AIDS, as Nef expression is an important requisite for efficient HIV-1 replication in the infected host cells, which suggests that it is a valid target for antiretroviral activity.

6. ...studies have shown (Nef) to be necessary for enhancing viral loadassociated infection, and inhibiting infected cell death."

\section{The multifarious activities of Nef in taking over host cells}

Nef is thought to increase the basal state of T-cell activation, and it may also inhibit or activate signaling events. A combination of these effects helps Nef to prevent premature

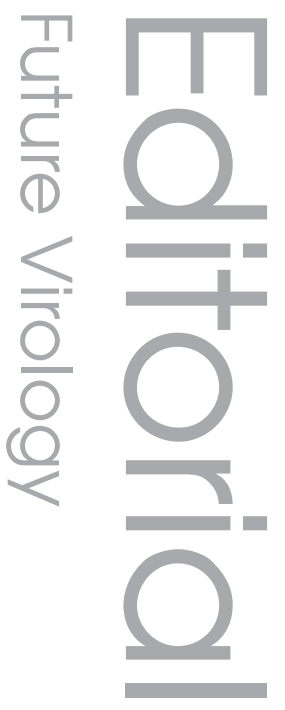

\section{Keywords}

- apoptosis $\approx \mathrm{CD} 4$ = HIV-1

- host cellular proteins - MHC-I downregulation $=$ Nef

- p21-activated kinase 2 - p53

tumor suppressing protein

- T-cell activation

Future : Medicine part of 


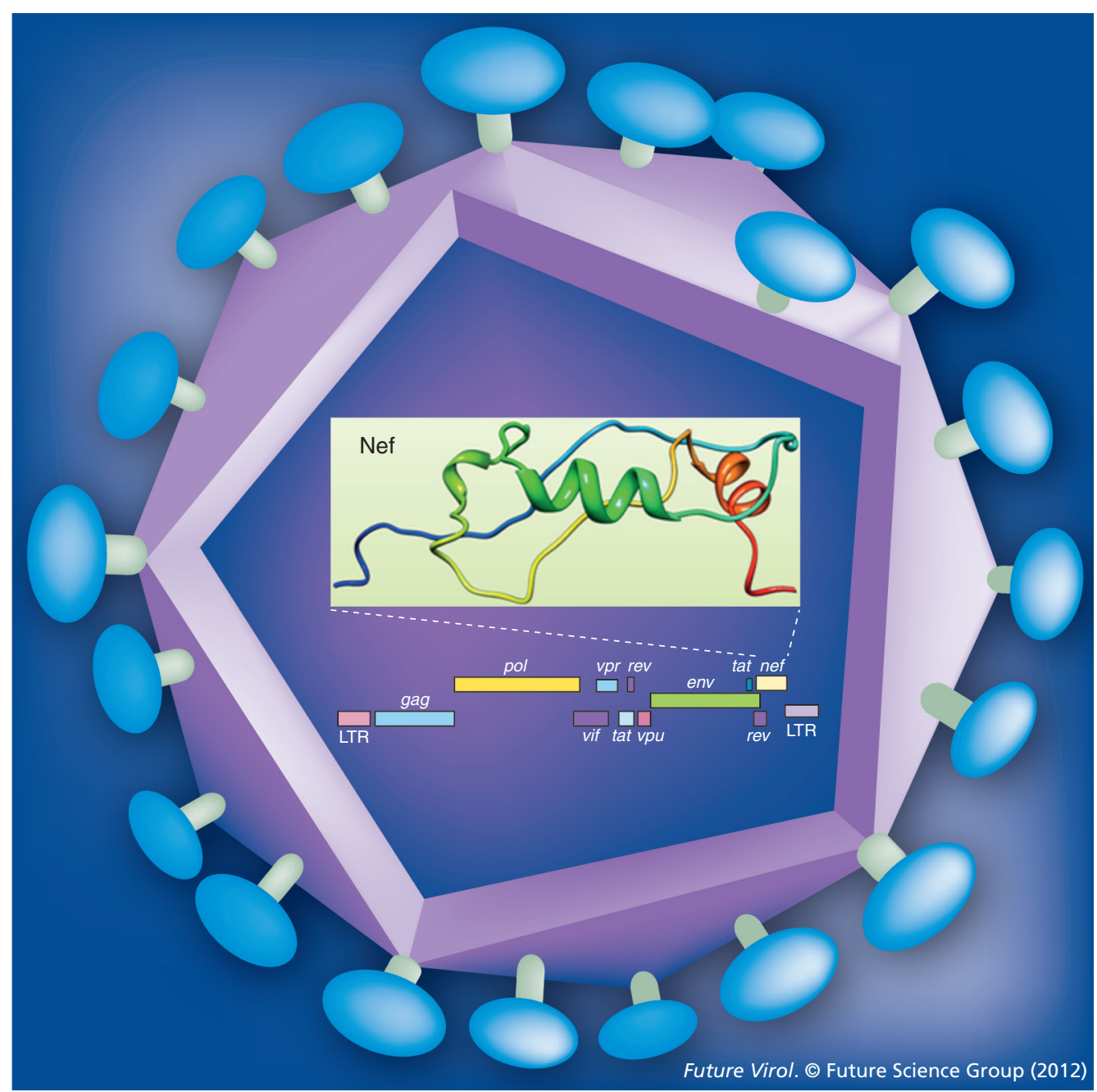

Figure 1. Schematic representation of the structure of HIV (outside), exhibiting its genome and Nef protein (inside).

activation-induced death of infected cells while simultaneously elevating their eligibility for HIV-1 replication. Nef increases the rate of CD4 endocytosis and lysosomal degradation. Extensive studies done on Jurkat T-cell lines showed that Nef expression negatively affects the induction of NF- $\kappa \mathrm{B}$. However, results obtained in Nef transgenic mice showed that Nef elevates T-cell signaling, which supports the evidence that it has pleiomorphic effects on T-cell activation that totally depend on the circumstances of expression [8]. Nef stimulates HIV infectivity, as virions produced in the presence of Nef, which is then packed into them and later cleaved by viral proteases, are ten-times as infectious compared with Nef-depleted virions [9].

In HIV-1-infected macrophages, Nef induces the release of soluble factors (sICAM-1 and sCD23), which stimulate the B-cell-mediated process of changing non-cycling $\mathrm{T}$ cells into cells permissive for enhancing viral infection and increasing the substrate lymphocyte population [10]. Nef co-expresses with endogenous Hck (tyrosine kinase) and leads to cellular transformation that activates myeloid lineage. Nef has a specific role in internalization of CD28 co-stimulatory molecule, which is necessary for T-cell activation. It restricts the interaction of Nef-expressing $\mathrm{T}$ cells with APCs, which in turn hastens viral spread due to the enhanced movement of infected T cells [11].

\section{Arresting cell migration}

Nef has also been reported to inhibit cell motility by phosphorylating the cofilin (conserved actin-depolymerizing factor), which is required in unphosphorylated form, and hence altering cellular kinase Pak2 function [12], which promotes motility, thereby indirectly restricting the T-lymphocyte migration. This serves as a valid strategy to invade the human immune system [13]. 


\section{Induction of apoptosis: a smart move}

HIV-1 Nef induces apoptosis in infected immune cells via the expression of Fas (CD95) and Fas ligand (CD95L), and Nef-induced CD95Lmediated killing of uninfected cells has also been reported. CD95L helps in evasion of the immune system by causing the apoptosis of HIV-specific CTLs. Association of Nef with ASK1 enhances the ability of Nef to alter the intracellular environment of infected host cells by enhancing their resistance to Fas- and TNF $\alpha$-mediated apoptosis [14]. One of the most interesting properties of $\mathrm{Nef}$ is its interaction with the $\mathrm{p} 53$ tumor-suppressing protein via its $\mathrm{N}$-terminus, which destabilizes $\mathrm{p} 53$, leading to a decrease in its proapoptotic, transcriptional and DNA-binding activities, and indeed protecting HIV infected cells from undergoing p53-mediated apoptosis. Additionally, $\mathrm{Nef}$ protects the infected cell by blocking external death signals coming from CTLs via the inhibition of ASK1, and also internal death signals via the phosphorylation of $\mathrm{Bcl}-2$-associated death promoter protein [15].

“...Nef expression is an important requisite for efficient HIV-1 replication

in the infected host cells, which suggests that it is a valid target for antiretroviral activity."

\section{Anticipated drug targets}

When considering Nef-based vaccines, CD4 might serve as the best target to be disrupted by an antiviral, whereas MHC-1 downregulation might not be as good an option, because blocking this function does not impact pathogenesis significantly. Insufficient knowledge about Pak2 activation may be one of the hindrances to using $\mathrm{Nef}$ as a popular vaccine target. Novel approaches, including miRNAs produced in HIV-1-infected cells that suppress both Nef function and HIV virulence through RNAi pathway, are also on the list.

\section{References}

1. Diskin R, Scheid JF, Marcovecchio PM et al. Increasing the potency and breadth of an HIV antibody by using structure-based rational design. Science 334(6060), 1289-1293 (2011).

2. O’Neill E, Kuo LS, Krisko JF, Tomchick DR, Garcia JV, Foster JL. Dynamic evolution of the human immunodeficiency virus type 1 pathogenic

\section{Necessity of the hour: Frequent Operation in Racing against Cruel Nef-mediated Egress (FORCE)}

Nef has been shown by various studies as one of the most important factors helping HIV to hack the cell machinery, but it is still not a perfect helper. A necessary characteristic for a hacker is to be unidentifiable, but Nef lacks this. Despite having so many properties, Nef is readily identifiable, and this shortcoming may be well used against it. Several novel epitopes have been found [SAXENA SK ETAL., Unpublished Data], which might serve as potential drug targets, and alternative strategies to conquer hacking of the cells and control mechanisms in Nef-mediated pathways should be devised. But all this is not a one-man show, and the current need is for a global, united effort. Interdisciplinary research efforts should be more encouraged, and synchronization between virologists, molecular biologists, system biologists, biochemists, immunologists, industry experts, medical intellectuals and policy makers is most desirable. This basic research needs a globally applied platform to face the problem, which may be provided with the involvement of in silico research approaches that may help in forecasting the future of designed vaccine candidates, as several vaccines tend to fail in clinical trials due to lack of proper understanding. Synthesis of novel research and re-evaluation of already-available data may one day be seen as a milestone in the battle against HIV. A great sense of urgency is required to address this matter.

\section{Financial \& competing interests disclosure \\ The authors are grateful to Council of Scientific and Industrial Research (CSIR-CCMB), India, for encour- agement and support for this work. An NIH Award (R37DA025576) also supports SK Saxena and MPN Nair. The authors have no other relevant affili- ations or financial involvement with any organization or entity with a financial interest in or financial conflict with the subject matter or materials discussed in the manuscript apart from those disclosed. \\ No writing assistance was utilized in the production of this manuscript.}

factor, Nef. J. Virol. 80 (3), 1311-1320 (2006).

3. Fackler OT, Alcover A, Schwartz O. Modulation of the immunological synapse: a key to HIV-1 pathogenesis? Nat. Rev. Immunol. 7(4), 310-317 (2007).

4. Fackler OT, Lu X, Frost JA et al. p21activated kinase 1 plays a critical role in cellular activation by Nef. Mol. Cell. Biol. 20 (7), 2619-2627 (2000).
5. Greenway AL, McPhee DA, Allen K et al. Human immunodeficiency virus type 1 Nef binds to tumor suppressor p53 and protects cells against $\mathrm{p} 53$-mediated apoptosis. J. Virol. 76(6), 2692-2702 (2002).

6. Collins KL, Chen BK, Kalams SA, Walker BD, Baltimore D. HIV-1 Nef protein protects infected primary cells against killing by cytotoxic T lymphocytes. Nature 391(6665), 397-401 (1998). 


\section{Editorial Saxena, Shrivastava, Tiwari \& Nair}

7. Schwartz O, Maréchal V, Le Gall S, Lemonnier F, Heard JM. Endocytosis of major histocompatibility complex class I molecules is induced by the HIV-1 Nef protein. Nat. Med. 2(3), 338-342 (1996).

8. Schindler M, Münch J, Kutsch O et al. Nef-mediated suppression of $\mathrm{T}$ cell activation was lost in a lentiviral lineage that gave rise to HIV-1. Cell 125(6), 1055-1067 (2006).

9. Malim MH, Emerman M. HIV-1 accessory proteins - ensuring viral survival in a hostile environment. Cell Host Microbe 3(6), 388-398 (2008).
10. Pomerantz RJ. HIV: cross-talk and viral reservoirs. Nature 424(6945), 136-137 (2003).

11. Swingler S, Mann A, Jacqué J et al. HIV-1 Nef mediates lymphocyte chemotaxis and activation by infected macrophages. Nat. Med. 5(9), 997-1003 (1999).

12. Stolp B, Reichman-Fried M, Abraham L et al. HIV-1 Nef interferes with host cell motility by deregulation of Cofilin. Cell Host Microbe 6(2), 174-186 (2009).

13. Swingler S, Brichacek B, Jacque JM, Ulich C, Zhou J, Stevenson M. HIV-1 Nef intersects the macrophage CD40L signalling pathway to promote resting-cell infection. Nature 424(6945), 213-219 (2003).

14. Geleziunas R, Xu W, Takeda K, Ichijo H, Greene WC. HIV-1 Nef inhibits ASK1dependent death signalling providing a potential mechanism for protecting the infected host cell. Nature 410 (6830), 834-838 (2001).

15. Wolf D, Witte V, Laffert B et al. HIV-1 Nef associated PAK and PI3-kinases stimulate Akt-independent Bad-phosphorylation to induce anti-apoptotic signals. Nat. Med. 7(11), 1217-1224 (2001). 